\title{
Coincidence of mutations in different connexin genes in Hungarian patients
}

\author{
TÍMEA TÓTH ${ }^{1}$, SUSAN KUPKA ${ }^{2,5}$, BIRGIT HAACK ${ }^{2,6}$, FERENC FAZAKAS $^{3}$, LáSZLÓ MUSZBEK ${ }^{3}$, \\ NIKOLAUS BLIN ${ }^{4}$, MARKUS PFISTER $^{2}$ and ISTVÁN SZIKLAI ${ }^{1}$ \\ ${ }^{1}$ Department of Otolaryngology, Medical and Health Science Center, University of Debrecen, Hungary; ${ }^{2}$ Department of \\ Otolaryngology, University Hospital Tübingen, Tübingen, Germany; ${ }^{3}$ Clinical Research Center, Haemostasis, Thrombosis \\ and Vascular Biology Research Group of the Hungarian Academy of Sciences, Medical and Health Science Center, \\ University of Debrecen, Hungary; ${ }^{4}$ Institute of Human Genetics, University of Tübingen, Tübingen, Germany
}

Received February 27, 2007; Accepted April 2, 2007

\begin{abstract}
Mutations in the GJB2 gene are the most common cause of hereditary prelingual sensorineural hearing impairment in Europe. Several studies indicate that different members of the connexin protein family interact to form gap junctions in the inner ear. Mutations in different connexin genes may accumulate and, consequently lead to hearing impairment. Therefore, we screened 47 Hungarian GJB2heterozygous (one mutation in coding exon of the GJB2 gene) patients with hearing impairment for DNA changes in two further connexin genes (GJB6 and GJB3) and in the $5^{\prime}$ non-coding region of $G J B 2$ including the splice sites. Eleven out of 47 GJB2-heterozygous patients analyzed carried the splice site mutation $-3170 \mathrm{G}>\mathrm{A}$ in the $5{ }^{\prime} \mathrm{UTR}$ region of $G J B 2$. One out of these 11 patients showed homozygous $-3170 \mathrm{G}>\mathrm{A}$ genotype in combination with p.R127H. Next to the GJB2 mutations we noted 2 cases of deletion in GJB6 [ $\triangle(G J B 6-$ D13S1830)] and 3 (2 new and 1 described) base substitutions in $G J B 3$ [c.357C>T, c.798C $>\mathrm{T}$ and c.94C $>\mathrm{T}$ (p.R32W)] which are unlikely disease-causing. Our results suggest the importance of routine screening for the rather frequent $3170 \mathrm{G}>\mathrm{A}$ mutation (in addition to c.35delG) in patients with hearing impairment.
\end{abstract}

Correspondence to: Dr Tímea Tóth, Department of Otolaryngology, Medical and Health Science Center, University of Debrecen, Nagyerdei krt. 98, 4012 Debrecen, Hungary

E-mail: ttimi@dote.hu

Present addresses: ${ }^{5}$ Department of General, Visceral and Transplant Surgery, University Hospital of Tübingen, Germany; ${ }^{6}$ Department of Molecular Pathology, University Hospital of Tübingen, Germany

Key words: hearing impairment, heterozygous GJB2 mutation, GJB2-splice site mutation, GJB3, GJB6

\section{Introduction}

Hearing impairment (HI) represents the most frequent neurosensory disorder worldwide. Contrary to former assumptions ascribing the basis of this highly heterogenous disease mainly to the ageing process and external factors, in recent years it has been demonstrated that at least $2 / 3$ of all hearing deficiencies are of genetic origin. Approximately $70 \%$ of all hereditary hearing impairment cases occur as non-syndromic forms without additional clinical symptoms. GJB2 is the gene responsible for a large proportion of these diseases (OMIM 121011). The GJB2 gene encodes the gap junction protein connexin 26 (CX26) which is expressed in the cochlea and may play a role in $\mathrm{K}^{+}$circulation between different partitions in the cochlea $(1,2)$. Gap junctions comprise the major system of intercellular communication for electrolytes, second messengers and metabolites. Therefore, it is not surprising that alterations in several members of the connexin protein family contribute to the development of hearing impairment, e.g. connexin 26, 30 and 31 (3-7).

Currently, more than 100 different mutations have been described within GJB2 making it the most frequently examined gene in patients with hearing impairment (8). Although major progress has been made over the last several years in understanding GJB2-based hearing impairment, various patients belonging to multiple ethnic groups were described with only one single heterozygous GJB2 mutation. The pathogenic mutations in this gene are generally inherited recessively and therefore induce hearing impairment only if occuring in both alleles; assessment and genetic counseling of single mutation carriers are difficult.

Several studies have demonstrated that most cells generally express more than one connexin isoform and assemble homomeric or heteromeric connexins leading to the formation of homotypic or heterotypic gap junctions (9-11). Four connexins (CX26, CX30, CX31 and CX43) were identified as prominently expressed in the cochlea. Moreover, digenic inheritance of mutations in two distinct connexin genes was shown to lead to hearing impairment (12). Therefore, we screened the patients with a heterozygous $G J B 2$ mutation for alterations in further connexin genes ( $G J B 3$ and 
GJB6) and for mutations in the basal GJB2 promoter as well as in the non-coding $G J B 2$ region.

\section{Materials and methods}

Subjects and selection criteria. In this study 410 patients were involved. Patients showed congenital, bilateral nonsyndromic sensorineural hearing impairment and were recruited from the outpatient service of the Department of Otolaryngology, University of Debrecen. The control group was composed of 156 unrelated individuals. Information on the medical history and pedigree structure was obtained in personal interviews with the affected individuals or with their unaffected relatives. Written informed consent was obtained from all participants and from parents of patients younger than 18 years. Control cases were collected comprising 156 individuals with normal hearing also originating from Hungary. The study was approved by the Ethics Committee of the University of Debrecen.

Case histories were obtained using a questionnaire regarding the following aspects, with special attention to disease: age at onset of SNHI, hearing aids, symmetry of the hearing impairment, middle ear infections, medical treatment, noise damage, trauma, meningitis, ototoxic agents, tinnitus, vertigo and other clinical manifestations (neurologic, ophthalmologic, gynecologic, pediatric, dermatologic, and orthopedic) to exclude other syndromic forms of hearing impairment.

Audiological methods. All patients involved in the study underwent otoscopic and audiometric examinations by using age-appropriate methods. We used air- and bone-conduction at $125,250,500,1000,2000,4000$, and $8000 \mathrm{~Hz}$ for all affected participants. The audiometric configuration, assessment of age at onset, severity and pattern of hearing loss by pure-tone audiometry, tympanometry and auditory brainstem response were performed according to the recommendations of the European Workgroup on Genetics of Hearing Impairment (13).

Genetic analysis. Anticoagulated venous blood (6 ml) was obtained from all tested individuals, and DNA was extracted according to the manufacurer's recommendations (Puregene kit, GENTRA System, Minneapolis, MN, USA).

GJB2 analysis. The coding exon of GJB2 was sequenced using a capillary sequencer model CEQ 8000 (Beckman Coulter) (14). Sequences were compared with the reference sequence (GenBank no. AF281280) using the DNAsis software (MWG).

Mutation c.380G >A (p.R127H) was screened in control individuals applying a restriction fragment length polymorphism (RFLP) assay. Using primers GJB2,3F (gtggctac cggagacatgag) and GJB2,5R (gggcaatgcgttaaactggc) a 409-bp PCR product was amplified. The mutation abolishes a restriction site for $S f a$ NI resulting in three bands (143, 189 and $77 \mathrm{bp})$ for the mutant and four bands (107, 36, 189 and $77 \mathrm{bp})$ for wild-type alleles after SfaNI digestion and polyacrylamide gel electrophoresis.

The 5' non-coding region of GJB2, promoter region -3458 to -3331 relative to the A of the ATG translation initiation codon located in exon 2 (15), and the non-coding first exon including both splice sites were sequenced using primers GJB2P,H2.1F (cagggcgctgggggcacttgggg) and GJB2P,H2.1R (caaccgctctgggtctcgcggtcc). Sequences were aligned with the reference sequence (Gen Bank no. U43932.1) utilizing the CEQ software (Beckman Coulter). The splice site mutation $-3170 \mathrm{G}>\mathrm{A}$ (IVS1(+1)G-->A) was screened by an RFLP assay. Applying primers FOR (ggtgtggggtgcggttaaaaggcg) and MUT_REV (cagtccggggccggcggggtca) a 221-bp PCR fragment was generated. Since the mutation destroys a restriction site for Eco91I, wild-type alleles show two bands (199 and $22 \mathrm{bp}$ ) and mutated alleles only one band (221 bp) after Eco91I digestion. Samples revealing a mutated allele were sequenced on both strands.

GJB3 analysis. The coding exon of GJB3 was sequenced using primers described elsewhere $(16,17)$. Sequences were compared with the reference sequence (GenBank no. NT_079620) using the CEQ software (Beckman Coulter).

The c.94C>T (p.R32W) sequence variant was screened applying primers $1 \mathrm{f}$ (acctattcattcatacgatgg) and 1r (gagtgtgca gcaggtagagg) (16). The variant has just one restriction site for $B c n I$ while the wild-type allele has two. After digestion and elecrophoresis, two DNA fragments (240 and $282 \mathrm{bp}$ ) for the mutant and three fragments $(79,161$ and $282 \mathrm{bp})$ for the wild-type allele were observed.

GJB6 analysis. Applying a multiple PCR strategy the 342-kb deletion $[\Delta(G J B 6-D 13 S 1830)]$ was examined. Using primers for1 (gccatgcatgtggcctacta), rev1 (actatctgaaatcagctcattc) and for2, del (cattgttgtgaactaacctcca, GenBank no. AL590096.16) a 441-bp PCR product was generated for wild-type alleles and a 480-bp fragment was produced for an allele representing the deletion.

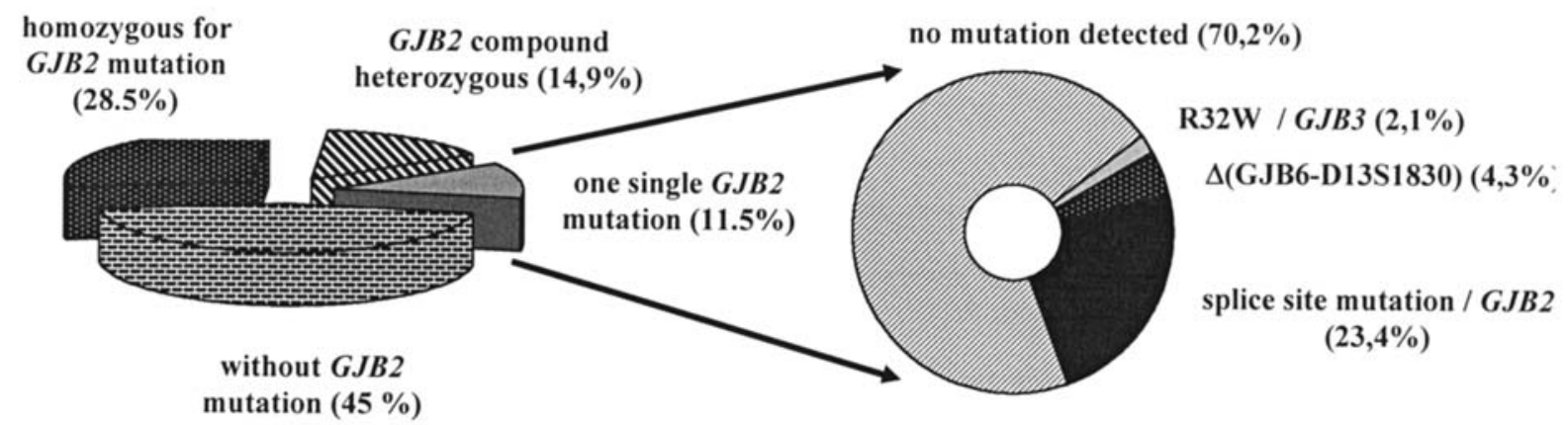

Figure 1. Distribution of GJB2 mutations detected in Hungarian hearing impaired patients and the frequency of further connexin mutations. 


\section{Results}

Audiologic. GJB2 mutations were detected in $225 / 410$ patients $(55 \%)$. All individuals were diagnosed with prelingual, sensorineural, bilateral, and moderate to profound hearing impairment. In most of the cases both ears presented a similar degree of hearing impairment. The auditory deficit involved all frequencies, and the morphology of the audiometric curves was flat in 153/225 (68\%) of the cases and slightly decreased towards the high frequencies in $72(32 \%)$ of the cases. No other configurations were observed.

GJB2 mutations. The most common GJB2 mutation in the Hungarian population is the c.35delG (18). The mutation frequency of c.35delG in this cohort was $39 \%(319 / 820$ alleles analyzed) which is in accordance with our previous studies (19). One hundred and seventeen patients showed the c.35delG mutation in GJB2 in homozygous form (28.5\%) and 61 were c.35delG compound heterozygous (14.8\%). Fortyseven patients $(11.4 \%)$ with sporadic hearing impairment showed only one GJB2 mutation (c.35delG, p.V27I, p.G59V, p.R127H or p.E129K) (Fig. 1 and Table I). Most of these patients $(24 / 47, \sim 51 \%)$ revealed the c.35delG mutation, whereas $3 / 47(6.4 \%)$ showed the c.79G $>$ A (p.V27I) mutation, $2 / 47$ (4.3\%) patients the c.385G $>A$ (p.E129K) and only one the c.177G $>$ T (p.G59V). A high number of the c.380G $>$ A (p.R127H) mutations were found $(17 / 47, \sim 36.2 \%)$ in our patients. Subsequently the c.380G $>$ A (p.R127H) DNA change revealed $4 / 156$ (allele frequency $1.3 \%$ ) heterozygous control individuals.

GJB2-non-coding region and promoter analysis. The first non-coding exon including the splice sites was analyzed for DNA changes. Ten heterozygous and one homozygous patient (11/47) showed the already described splice site mutation $-3170 \mathrm{G}>\mathrm{A}$ in the non-coding GJB2 region (23.4\%). No $-3170 \mathrm{G}>\mathrm{A}$ splice site mutation was found in 156 control individuals $(<0.32 \%$ allele frequency).

GJB6 mutations. All 47 GJB2-heterozygous patients were analyzed for the 342-kb deletion [ $\triangle(G J B 6-\mathrm{D} 13 \mathrm{~S} 1830)]$ using a multiple PCR strategy. Only two patients out of 47 GJB2heterozygous patients showed the deletion in heterozygous form, accounting for $\sim 4.3 \%$. This deletion [ $\triangle(G J B 6-$ D13S1830)] was analyzed in 46/185 hearing impaired patients without GJB2 mutations and the corresponding control samples as well. In contrast to other studies, none of the 46 patients negative for $G J B 2$ mutations carried this deletion. Furthermore, 156 controls exhibited only wild-type alleles.

GJB3 mutations. By sequencing the coding region of GJB3, 3 common GJB3 (2 new and 1 described) base substitutions (c.357C > T, c.798C > T, and p.R32W) were detected in our patients and the corresponding control samples, respectively. The new c.357C $>$ T, c.798C $>\mathrm{T}$ base changes were not the result of a change of amino acid and they were likely polymorphisms since they also occurred in the control population. The c.357C $>\mathrm{T}$ variant occured with an incidence of $\sim 6 \%$ (controls) to $15 \%$ (patients), a frequency comparable to other studies $(17,20)$. The SNP c.798C $>$ T occured in $6 \%$ (patients) to $11 \%$ (controls) of all analyzed individuals, which also concurs with other research (20). In addition the c. $94 \mathrm{C}>\mathrm{T}$ (p.R32W) variant in GJB3 was detected in one out of 47 heterozygous patients $(2.1 \%)$. This DNA variant was the only GJB3 mutation resulting in a change of amino acid. Nine out of 156 control individuals (5.7\%) carried this DNA change in the heterozygous state.

\section{Discussion}

Mutations in GJB2, the gene encoding the gap-junction protein connexin 26, are the most common cause of recessively inherited prelingual hearing impairment in Europe. Since several studies have demonstrated that single mutations in different connexin genes lead to digenic hearing impairment, we investigated a patient cohort consisting of Hungarian patients with one heterozygous GJB2 mutation for mutations in further connexin genes and the regulatory region of $G J B 2$.

GJB2 promoter and the non-coding region. The basal GJB2 promoter was characterized by Tu and Kiang in 1998. Using reporter gene assays they localized the promoter region to position -3458 to -3331 relative to the translation initiation codon. Furthermore, a critical regulatory region extending from -3427 to -3399 was determined (15). Analyzing this DNA region via direct sequencing in GJB2-heterozygous Hungarian patients revealed no DNA change suggesting an infrequent involvement of promoter mutations in the development of hearing impairment in our patients.

In contrast, mutation $-3170 \mathrm{G}>\mathrm{A}$ which is located within the splice site, and therefore may have a pathogenic impact, was detected in $23.4 \%$ of GJB2-heterozygous patients (Table I). It is likely that the $-3170 \mathrm{G}>\mathrm{A}$ allele is either not transcribed or the mRNA is unstable, thereby leading to hearing impairment. This recessive mutation occurs most frequently in combination with c.35delG resulting in severe to profound hearing impairment $(21,22)$. Less common is the occurrence in conjunction with GJB2 mutations p.R184P and c.167delT (23-25). Our new observation for the Hungarian population is similar. Ten heterozygous $-3170 \mathrm{G}>\mathrm{A}$ carriers also had the c. 35 delG mutation, whereas only one individual had the p.R127H mutation in combination with homozygous splice site mutations. The severity of $\mathrm{HI}$ varies from moderate to profound in the cases with [GJB2: c.35delG] + [5'UTR GJB2: $-3170 \mathrm{G}>\mathrm{A}]$ genotype. The binaural mean pure tone average (PTA) threshold for air conduction at $0.5,1$ and $2 \mathrm{kHz}$ $\left(\mathrm{PTA}_{0.5,1,2 \mathrm{kHz}}\right)$ was moderate in two patients, severe in three and profound in five. The patient with the homozygous splice site mutation was a 3-year-old girl. Auditory brainstem response $(\mathrm{ABR})$ was performed and showed moderate $\mathrm{HI}$.

GJB6 and $\Delta($ GJB6-D13S1830) deletion. It is known that CX26 can form heteromeric and heterotypic channels with other inner ear connexins in mammals (26). Co-immunostaining showed expression of CX26 (GJB2) and CX30 (GJBO) in the same gap junction plaques $(27,28)$. Interaction of both proteins was demonstrated pointing to their ability to form heteromeric CX26/CX30 connexins in vivo (29). The 


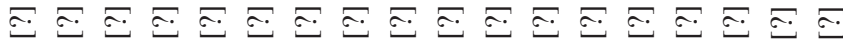

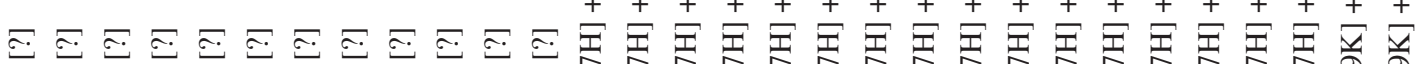

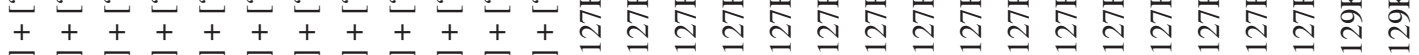

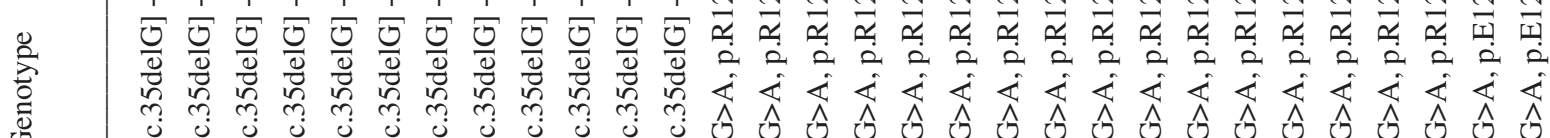

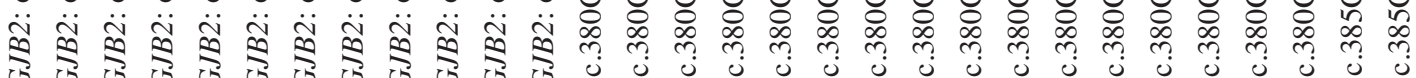

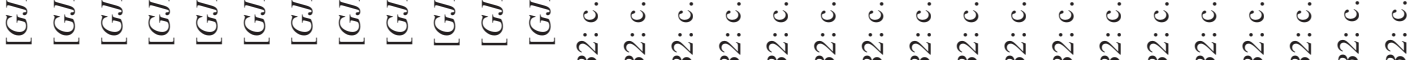
芯

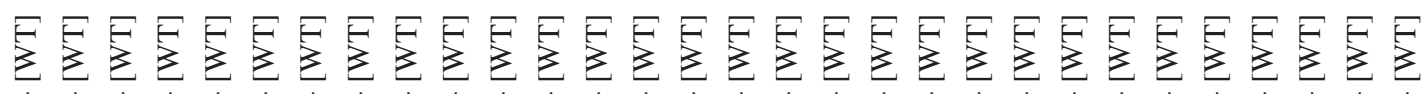
$++++++++++++++++++++++++++++++$

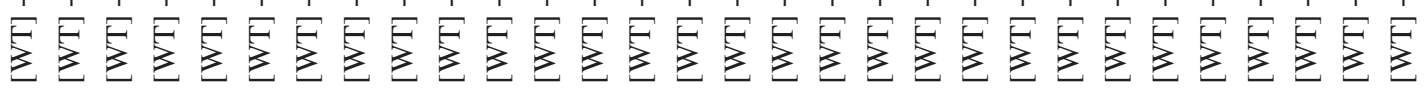

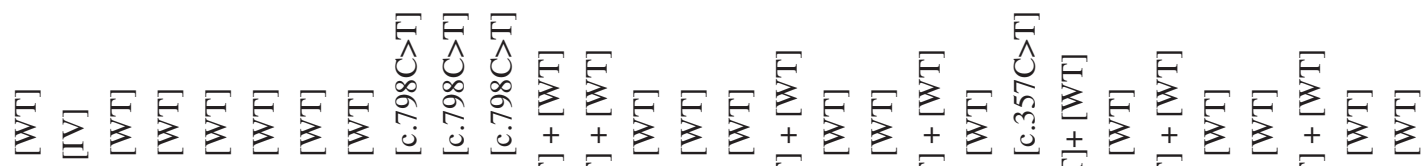

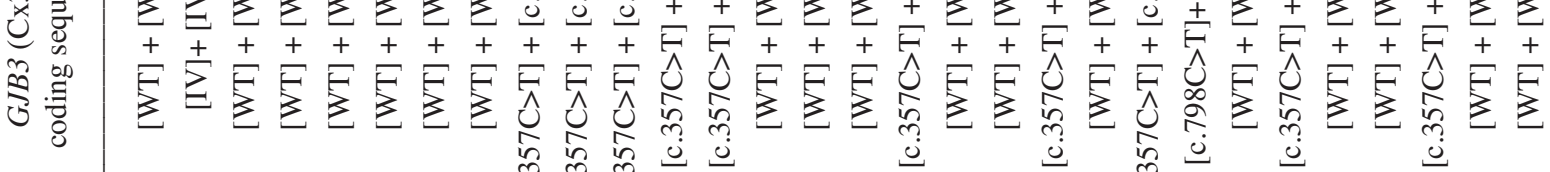
它 0 计

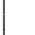

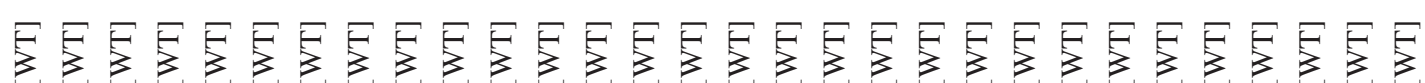

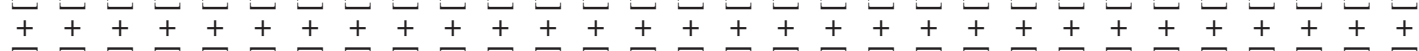

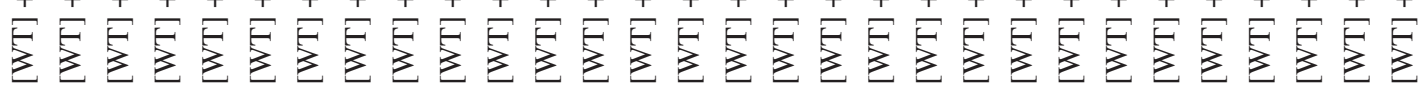

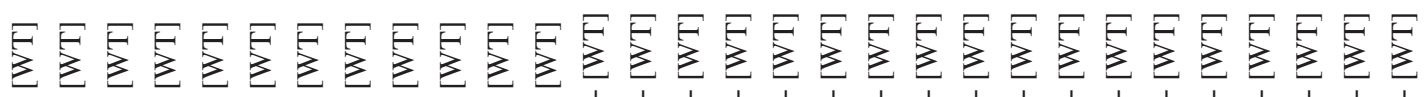

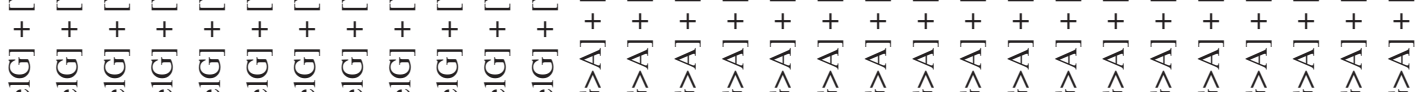

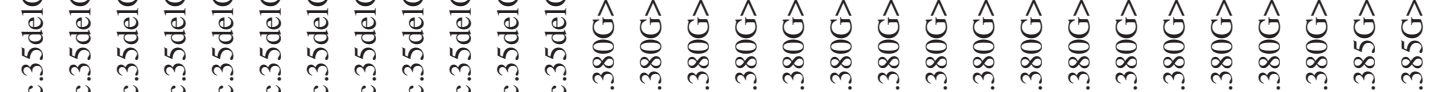

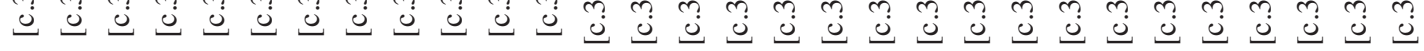




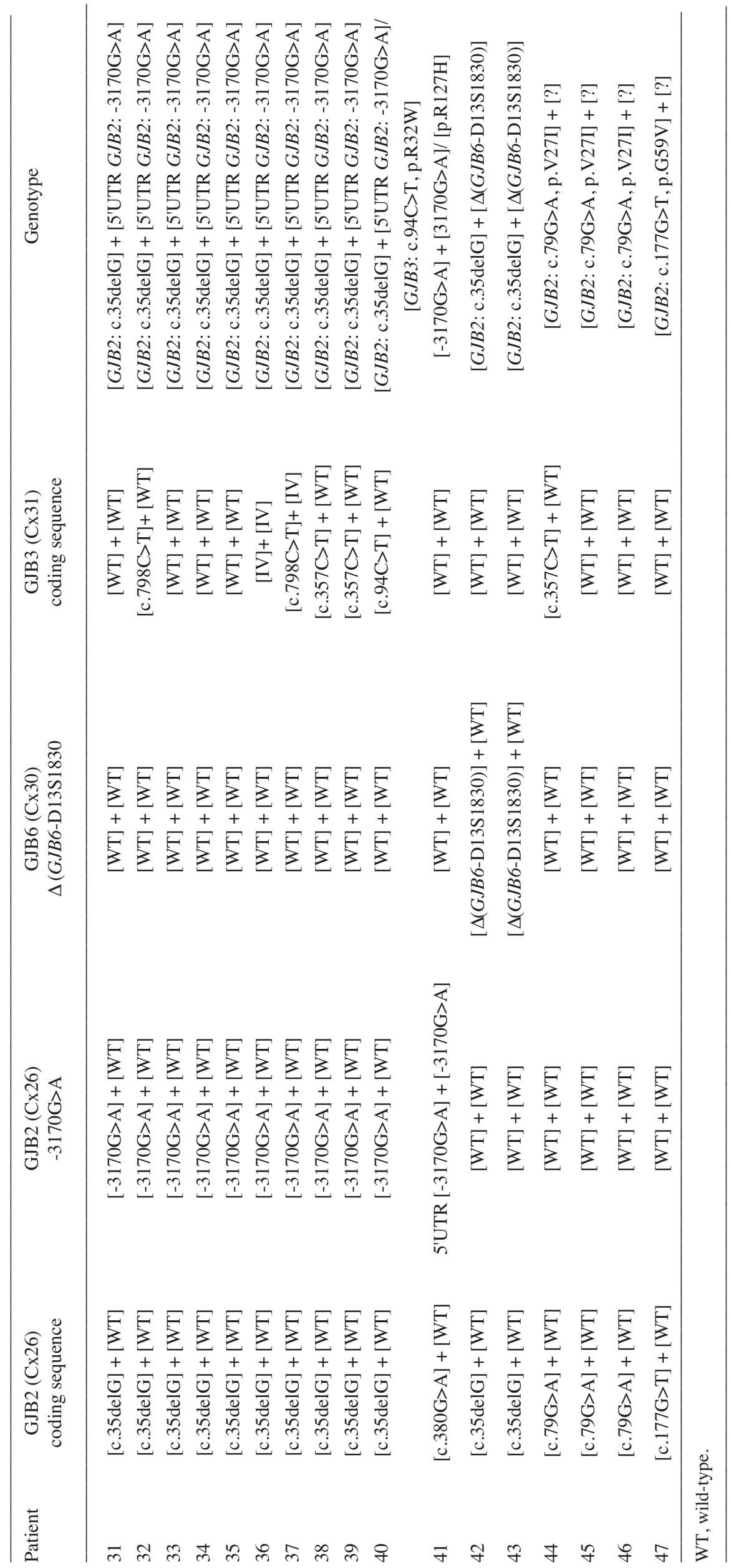


GJB6 situation is particular because of its chromosomal localization within $50 \mathrm{~kb}$ to GJB2. Mutations in GJB6 have been shown to result in non-syndromic autosomal dominant and recessive hearing impairment $(6,30)$. Recently, the impact of GJB6 in the development of hearing impairment was confirmed by the generation of a knock-out mouse model (31). These mice exhibited severe hearing impairment. The cochlear sensory epithelium started to degenerate by cell apoptosis after P18 resulting in the deterioration of hearing in adult animals (31). A common 342-kb spanning deletion $[\Delta(G J B 6-\mathrm{D} 13 \mathrm{~S} 1830)]$ was identified in patients who carried one heterozygous pathogenic GJB2 mutation (c.35delG) $(30,32,33)$ (Table I). These patients are thought to develop hearing impairment with variable severity due to a digenic pattern of inheritance (34). $\Delta(G J B 6-\mathrm{D} 13 \mathrm{~S} 1830)$ leaves the $G J B 2$ coding region intact but deletes a large region close to GJB2 and truncates GJB6. Thus, the deletion may eliminate an upstream regulatory element for $G J B 2$ that is essential for the normal expression of this gene in the inner ear. This deletion is mainly found in combination with a GJB2 mutation, and the associated $\mathrm{HI}$ is assumed to be caused either by the deletion of a putative $G J B 2$ regulatory element or by digenic inheritance $(12,33)$. Our two patients with the c.35delG mutation combined with the $\Delta$ (GJB6-D13S1830) mutation showed more severe hearing impairment compared to the other patients, whereas the group of patients combining the c. 35 delG with the splice site mutation $-3170 \mathrm{G}>\mathrm{A}$ showed significantly less severe hearing loss. This result is consistent with the multicenter study of Snoeckx and coworkers (35). The frequency of the 342-kb deletion [ $\Delta$ (GJB6-D13S1830)] affecting $G J B 6$ varies tremendously between different populations. In our study, as well as in other studies of Polish and Austrian patients, the deletion was observed infrequently $(36,37)$. In contrast, a multicenter study in nine countries revealed high mutation frequencies with the highest ones (32-72\%) observed in France, Spain, Israel, and the UK (33). Furthermore, the frequency of a digenic cause for hearing impairment based on GJB2/[ $\triangle(G J B 6$-D13S1830)] mutations in North American patients varied between 16 and $20 \%$ (38-40). According to our results, mutations within the coding region of GJB6 and the 342-kb deletion encompassing parts of GJB6 seem not to be a common cause for hearing impairment in Hungarian patients.

GJB3 sequence variants. In several studies mutations within GJB3 were described which resulted in HI $(5,17,20,41)$. In a family with palmoplantar keratoderma and various forms of $\mathrm{HI}$, Kelsell and coworkers were the first to detect the p.R32W mutation which occurred in combination with two missense mutations in GJB2 (p.M34T and p.D66H) segregating with the skin disease (41). Since p.R32W affects a highly conserved residue, the authors supposed that the high frequency $\mathrm{HI}$ observed in their patients resulted from defective CX31/ CX26 channel formation caused by additive mutations in both genes. In contrast, Lopes-Bigas and coworkers demonstrated that p.R32W in GJB3 did not segregate with HI and/or skin disease in their Spanish patient cohort (42). They presented a mother of a patient who carried c.35delG $(G J B 2)$ in combination with p.R32W (GJB3) and showed normal hearing and absence of skin disease. The authors presumed that p.R32W (GJB3) most likely represents a polymorphism with a frequency of $7.5 \%$ in the Spanish population. Rouan and coworkers subsequently showed that the intracellular coupling of HeLa cells transfected with R32W-CX31 was comparable to wild-type CX31, which is consistent with the view that R32W is a polymorphism of CX31 (43). In our study p.R32W was the only mutation that caused an amino acid exchange found in our GJB2-heterozygous patients (Table I). Only one patient, who was compound heterozygous for c. $35 \mathrm{delG}$ and the splice site mutation $-3170 \mathrm{G}>\mathrm{A}$ in GJB2, displayed this mutation. Since $5.7 \%$ of the Hungarian control individuals analyzed also showed this DNA change, it most likely represents a common polymorphism.

In summary, approximately one fourth of all patients with one heterozygous GJB2 mutation carried another mutation in the 5'UTR region of $G J B 2$ (splice site mutation $-3170 \mathrm{G}>\mathrm{A}$ ). RFLP analysis as presented in this study provides an efficient tool to analyze patients with one heterozygous GJB2 mutation at a relatively low cost. Therefore, we suggest the importance of the additional routine screening for the $-3170 \mathrm{G}>\mathrm{A}$ mutation in $G J B 2$-heterozygous hearing impaired patients. Further mutations in the GJB3 and GJB6 genes are less frequently responsible for the hereditary hearing impairment in our patients.

\section{Acknowledgements}

This study was supported in part by grants of the ElseKröner-Fresenius-Stiftung, the fortüne programme (1028-0-0) Tübingen, the European Commission, Marie Curie Training Site, HEARING (QLG3-CT-2001-60009); and the Medical and Health Science Center University of Debrecen, Mecenatura Grant (16/2005), the Hungarian National Research Fund (OTKA-K63743), and the ETT 206/2006. We thank Guy van Camp for providing a DNA sample revealing the $342-\mathrm{kb}$ deletion affecting GJB6, and are grateful to Brigitta BoddenKamps for the technical assistance.

\section{References}

1. Kikuchi T, Adams JC, Paul DL and Kimura RS: Gap junction systems in the rat vestibular labyrinth: immunohistochemical and ultrastructural analysis. Acta Otolaryngol 114: 520-528, 1994.

2. Kikuchi T, Kimura RS, Paul DL and Adams JC: Gap junctions in the rat cochlea: immunohistochemical and ultrastructural analysis. Anat Embryol 191: 101-118, 1995.

3. Denoyelle F, Weil D, Maw MA, Wilcox SA, Lench NJ, Allen-Powell DR, Osborn AH, et al: Prelingual deafness: high prevalence of a 30 delG mutation in the connexin 26 gene. Hum Mol Genet 6: 2173-2177, 1997.

4. Kelsell DP, Dunlop J, Stevens HP, Lench NJ, Liang JN, Parry $G$ and Mueller RF: Connexin 26 mutations in hereditary non-syndromic sensorineural deafness. Nature 387: 80-83, 1997.

5. Xia JH, Liu CY, Tang BS, Pan Q, Huang L, Dai HP, Zhang BR, et al: Mutations in the gene encoding gap junction protein beta3 associated with autosomal dominant hearing impairment. Nat Genet 20: 370-373, 1999.

6. Grifa A, Wagner CA, D'Ambrosio L, Melchionda S, Bernardi F, Lopez-Bigas N, Rabionet R, et al: Mutations in GJB6 cause nonsyndromic autosomal dominant deafness at DFNA3 locus. Nat Genet 23: 16-18, 1999.

7. Liu XZ, Xia XJ, Xu LR, Pandya A, Liang CY, Blanton SH, Brown SD, Steel KP and Nance WE: Mutations in connexin31 underlie recessive as well as dominant non-syndromic hearing loss. Hum Mol Genet 9: 63-67, 2000. 
8. Connexins and deafness homepage. World wide web URL: http://davinci.crg.es/deafness.

9. Bukauskas FF, Elfgang C, Willecke $\mathrm{K}$ and Weingart R: Heterotypic gap junction channels (connexin26-connexin32) violate the paradigm of unitary conductance. Pflugers Arch 429: 870-872, 1995

10. He DS, Jiang JX, Taffet SM and Burt JM: Formation of heteromeric gap junction channels by connexins 40 and 43 in vascular smooth muscle cells. Proc Natl Acad Sci USA 96: 6495-6500, 1999.

11. Martin PE, Blundell G, Ahmad S, Errington RJ and Evans WH: Multiple pathways in the trafficking and assembly of connexin 26,32 and 43 into gap junction intercellular communication channels. J Cell Sci 114: 3845-3855, 2001.

12. Pallares-Ruiz N, Blanchet P, Mondain M and Roux AF: A large deletion including most of GJB6 in recessive non-syndromic deafness: a digenic effect? Eur J Hum Genet 10: 72-76, 2002.

13. European Work Group on Genetics of Hearing Impairment. Info letter 2: Nov., 1996.

14. Kupka S, Braun S, Aberle S, Haack B, Ebauer M, Zeissler U, Zenner HP, et al: Frequencies of GJB2 mutations in German control individuals and patients showing sporadic nonsyndromic hearing impairment. Hum Mutat 20: 77-78, 2002.

15. Tu ZJ and Kiang DT: Mapping and characterization of the basal promoter of the human connexin26 gene. Biochim Biophys Acta 1443: 169-181, 1998.

16. Lopez-Bigas N, Rabionet R, Martinez E, Banchs I, Arbones ML and Estivill X: Identification of seven novel SNPS (five nucleotide and two amino acid substitutions) in the connexin31 (GJB3) gene. Hum Mutat 15: 481-482, 2000.

17. Mhatre AN, Weld E and Lalwani AK: Mutation analysis of Connexin 31 (GJB3) in sporadic non-syndromic hearing impairment. Clin Genet 63: 154-159, 2003.

18. Tóth T, Kupka S, Sziklai I, Zenner HP, Blin N and Pfister M: Frequency of the recessive 30 delG mutation in the GJB2 gene in Northeast-Hungarian individuals and patients with hearing impairment. Int J Mol Med 8: 189-192, 2001

19. Tóth T, Kupka S, Haack B, Riemann K, Braun S, Fazakas F, Zenner HP, et al: GJB2 mutations in patients with nonsyndromic hearing loss from Northeastern Hungary. Hum Mutat 23: 631-632, 2004 .

20. Chu EA, Mhatre AN, Lustig LR and Lalwani AK: Implication of mutations in Connexin 31 in cochlear implant outcome. Gen Funct Dis 2: 214-220, 2001.

21. Denoyelle F, Marlin S, Weil D, Moatti L, Chauvin P, Garabedian EN and Petit C: Clinical features of the prevalent form of childhood deafness, DFNB1, due to a connexin-26 gene defect: implications for genetic counselling. Lancet 353: 12981303,1999

22. Marlin S, Garabedian EN, Roger G, Moatti L, Matha N, Lewin P, Petit $\mathrm{C}$ and Denoyelle F: Connexin 26 gene mutations in congenitally deaf children: pitfalls for genetic counseling. Arch Otolaryngol 127: 927-933, 2001.

23. McGuirt WT, Prasad SD, Cucci RA, Green GE and Smith RJ: Clinical presentation of DFNB1. Adv Otorhinolaryngol 61: 113-119, 2002

24. Najmabadi H, Cucci RA, Sahebjam S, Kouchakian N and Smith RJ: GJB2 mutations in Iranians with autosomal recessive non-syndromic sensorineural hearing loss. Hum Mutat 19: 572, 2002.

25. Orzan E, Murgia A, Polli R, Martella M, Mazza A, Zacchello F and Babighian G: Connexin 26 preverbal hearing impairment: mutation prevalence and heterozygosity in a selected population. Int J Audiol 41: 120-124, 2002

26. Forge A, Becker D, Casalotti S, Edwards J, Marziano N and Nevill G: Gap junctions in the inner ear: comparison of distribution patterns in different vertebrates and assessement of connexin composition in mammals. J Comp Neurol 8;467(2): 207-231, 2003
27. Lautermann J, ten Cate WJ, Altenhoff P, Grummer R, Traub O, Frank H, Jahnke $\mathrm{K}$ and Winterhager E: Expression of the gapjunction connexins 26 and 30 in the rat cochlea. Cell Tissue Res 294: 415-420, 1998

28. Ahmad S, Chen S, Sun J and Lin X: Connexins 26 and 30 are co-assembled to form gap junctions in the cochlea of mice. Biochem Biophys Res Commun 307: 362-368, 2003.

29. Marziano NK, Casalotti SO, Portelli AE, Becker DL and Forge A Mutations in the gene for connexin 26 (GJB2) that cause hearing loss have a dominant negative effect on connexin 30. Hum Mol Genet 12: 805-812, 2003.

30. Lerer I, Sagi M, Ben Neriah Z, Wang T, Levi H and Abeliovich D: A deletion mutation in GJB6 cooperating with a GJB2 mutation in trans in non-syndromic deafness: A novel founder mutation in Ashkenazi Jews. Hum Mutat 18: 460, 2001

31. Teubner B, Michel V, Pesch J, Lautermann J, Cohen-Salmon M, Sohl G, Jahnke K, et al: Connexin30 (Gjb6)-deficiency causes severe hearing impairment and lack of endocochlear potential. Hum Mol Genet 12: 13-21, 2003.

32. Del Castillo I, Villamar M, Moreno-Pelayo MA, Del Castillo FJ, Alvarez A, Telleria D, Menendez I and Moreno F: A deletion involving the connexin 30 gene in nonsyndromic hearing impairment. N Engl J Med 346: 243-249, 2002.

33. Del Castillo I, Moreno-Pelayo MA, Del Castillo FJ, Brownstein Z, Marlin S, Adina Q, Cockburn DJ, et al: Prevalence and evolutionary origins of the del(GJB6-D13S1830) mutation in the DFNB1 locus in hearing-impaired subjects: a multicenter study. Am J Hum Genet 73: 1452-1458, 2003.

34. Bolz H, Schade G, Ehmer S, Kothe C, Hess M and Gal A: Phenotypic variability of non-syndromic hearing loss in patients heterozygous for both c.35delG of GJB2 and the 342-kb deletion involving GJB6. Hear Res 188: 42-46, 2004.

35. Snoeckx RL, Huygen PL, Feldmann D and Camp GV: GJB2 mutations and degree of hearing loss: a multi-center study. Am J Hum Genet 77: 945-957, 2005.

36. Wiszniewska J, Wiszniewski $\mathrm{W}$ and $\mathrm{Bal} \mathrm{J}$ : The principles of molecular diagnosis of recessive forms of prelingual nonsyndromic hearing loss. Med Wieku Rozwoj 6: 309-318, 2002.

37. Gunther B, Steiner A, Nekahm-Heis D, Albegger K, Zorowka P, Utermann $\mathrm{G}$ and Janecke A: The 342-kb deletion in GJB6 is not present in patients with non-syndromic hearing loss from Austria. Hum Mutat 22: 180, 2003.

38. Pandya A, Arnos KS, Xia XJ, Welch KO, Blanton SH, Friedman TB, Garcia Sanchez G, et al: Frequency and distribution of GJB2 (connexin 26) and GJB6 (connexin 30) mutations in a large North American repository of deaf probands. Genet Med 5: 295-303, 2003.

39. Stevenson VA, Ito M and Milunsky JM: Connexin-30 deletion analysis in connexin-26 heterozygotes. Genet Test 7: 151-154, 2003 .

40. Wu BL, Kenna M, Lip V, Irons M and Platt O: Use of a multiplex PCR/sequencing strategy to detect both connexin 30 (GJB6) $342 \mathrm{~kb}$ deletion and connexin 26 (GJB2) mutations in cases of childhood deafness. Am J Med Genet 121A: 102-108, 2003.

41. Kelsell DP, Wilgoss AL, Richard G, Stevens HP, Munro CS and Leigh IM: Connexin mutations associated with palmoplantar keratoderma and profound deafness in a single family. Eur J Hum Genet 8: 469-472, 2000.

42. Lopez-Bigas N, Rabionet R, Arbones ML and Estivill X: R32W variant in Connexin 31: mutation or polymorphism for deafness and skin disease? Eur J Hum Genet 9: 70, 2001

43. Rouan F, Lo CW, Fertala A, Wahl M, Jost M, Rodeck U, Uitto J and Richard G: Divergent effects of two sequence variants of GJB3 (G12D and R32W) on the function of connexin 31 in vitro. Exp Dermatol 12: 191-197, 2003. 